\title{
DATOS SOBRE LA VEGETACIÓN DE ANDALUCÍA. III. SERIES DE VEGETACIÓN CARACTERIZADAS POR QUERCUS SUBER L.
}

\author{
Andrés V. PÉREZ LATORRE, José Ma . NIETO CALDERA y Baltasar CABEZUDO
}

\begin{abstract}
RESUMEN. Datos sobre la vegetación de Andalucía. III. Series de vegetación caracterizadas por Quercus suber $L$. Los alcornocales son una de las formaciones vegetales mas diversas y con mayor extensión de Andalucía. La presencia de Quercus suber es constante en ocho series o subseries de vegetación: Myrto communis-Querceto suberis S, Teucrio baetici-Querceto suberis S, Smilaci mauritanicae-Querceto rotundifoliae quercetoso suberis subsigmetum, Tamo communis-Oleeto sylvestris quercetoso suberis subsigmetum, Sanguisorbo hybridae-Querceto suberis S, Pyro bourgaeanae-Querceto rotundifoliae quercetoso suberis subsigmetum, Paeonio coriaceae-Querceto rotundifoliae quercetoso fagineae subsigmetum variante con Quercus suber, Adenocarpo decorticantis-Querceto rotundifoliae quercetoso suberis subsigmetum. Cada serie presenta un peculiar dinamismo en función de su uso ancestral, de su uso actual, de la variación de los factores ambientales y de su localización biogeográfica. Se describen en este trabajo dos nuevas subasociaciones: Ulici eriocladi-Cistetum ladaniferi cistetosum albidi y Phyllireo angustifoliae-Quercetum lusitanicae cytisetosum tribracteolati.
\end{abstract}

Palabras clave. Quercus suber, alcornocales, series de vegetación, Andalucía, España.

ABSTRACT. Data about andalusian vegetation. III. Vegetation series with Quercus suber L. presence. Quercus suber woods are one of the natural forests with more diversity and more expanse in Andalusia (Spain). Quercus suber is a dominant or common species in eight vegetation series or subseries: Myrto communis-Querceto suberis S, Teucrio baetici-Querceto suberis S, Smilaci mauritanicae-Querceto rotundifoliae quercetoso suberis subsigmetum, Tamo communis-Oleeto sylvestris quercetoso suberis subsigmetum, Sanguisorbo hybridae-Querceto suberis S, Pyro bourgaeanae-Querceto rotundifoliae quercetoso suberis subsigmetum, Paeonio coriaceae-Querceto rotundifoliae quercetoso fagineae subsigmetum, var. of Quercus suber, Adenocarpo decorticantis-Querceto rotundifoliae quercetoso suberis subsigmetum. These vegetation series show peculiar sucesional dynamism of their communities depending on their traditional use, environmental factors and biogeographical location. Two new subasociations, Ulici eriocladi-Cistetum ladaniferi cistetosum albidi and Phyllireo angustifoliae-Quercetum lusitanicae cytisetosum tribracteolati, are described in this paper.

Key words. Quercus suber woods, vegetation series, Andalusia, Spain.

\section{INTRODUCCIÓN Y OBJETIVOS}

El alcornocal es una formación vegetal directamente influida por el hombre. Su actual distribución, estructura y composición obede- cen a múltiples factores. En palabras de Pons y Thinon (1987) «los cambios inducidos (en la vegetación) son tan grandes, que un análisis de las presentes relaciones entre ecosistemas $y$ factores ambientales puede tener significa- 
do sólo si tiene en prioritaria consideración todos los pasados antropogénicos de los ecosistemas». No obstante parte de sus comunidades aún se encuentran en buen estado de conservación, de tal manera que todavía son susceptibles de ser interpretadas fitosociológicamente. El presente trabajo, continuación de los ya publicados por Nieto et al. (1990) y Pérez Latorre et al. (1993), tiene como objetivo principal el estudio de las series de vegetación caracterizadas por presentar como etapa boscosa a formaciones de Quercus suber. Para ello mencionamos las principales comunidades que las integran, los principales factores que afectan a su dinamismo y hacemos referencia al geosigmentum en donde se integra cada una de ellas.

Han sido numerosos los autores que han estudiado el área que nos ocupa y que hacen referencia al alcornocal o a sus etapas de sustitución; de ellos destacamos Ceballos y Vicioso (1933), Ceballos y Martín Bolaños (1930), Bellot (1945), Rivas Goday (1954, 1964b), Rivas Goday et al. (1962), Rivas Goday et al. (1965), Rivas Goday y Rivas Martínez (1971a, 1971b), Rivas Martínez (1974), Prieto y Espinosa (1975), Pérez Chiscano (1976), Achhal et al. (1979), Rivas Martínez (1979), Rivas Martínez et al. (1980), Barbero et al. (1981), Benabid (1984, 1994), Martínez Parras et al. (1987a, 1987b), Asensi y Díez Garretas (1987), Ladero (1987), Rivas Martínez (1987), Quézel et al. (1988), Díez et al. (1988), Ladero et al. (1990), Cano y Valle (1989), Nieto Caldera et al. (1990), Rivas Martínez et al. (1990) y Pérez Latorre et al. (1993).

\section{MATERIAL Y MÉTODO}

El área de estudio se localiza en Andalucía. Abarca a Sierra Morena, los depósitos arenosos del valle del Guadalquivir, Campo de Gibraltar y cordilleras litorales béticas. Litológicamente predominan los sustratos de naturaleza silícea, que se encuadran en cuatro grandes grupos: materiales del Campo de Gibraltar (areniscas y margoareniscas), materiales metamórfico-esquistosos (pizarras, cuarcitas, gneises, etc.), rocas plutónicas y volcánicas (granitos, dioritas, etc.) y rocas sedimentarias (brechas y calizas descarbonatadas, arenas, margas silíceas, gravas cuarzosas, etc.). Biogeográficamente seguimos el esquema establecido en Pérez Latorre et al. (1993). El encuadre bioclimático de cada serie se ha basado en el estudio de los datos recogidos en De León Llamazares (1989 a-h) de las estaciones climáticas situadas en el areal ocupado por cada una de ellas.

Para el estudio de las series de vegetación se ha utilizado el método sinfitosociológico (Rivas Martínez, 1976, 1985 y 1987; Gehú y Rivas Martínez, 1981). El texto del presente trabajo se estructura por series dentro de los complejos ambientales que constituyen los pisos bioclimáticos. Cada serie o subserie se comenta bajo los aspectos de variabilidad (faciaciones) y asociaciones que constituyen las fases dinámico-estructurales: vegetación arbórea (Quercetalia ilicis), preforestal arbustiva y herbácea vivaz (PistacioRhamnetalia, Cytisetalia y Origanetalia) y matorral (Lavanduletalia stoechidis, Stauracantho-Halimietalia commutati y Calluno-Ulicetalia) que ya fueron descritas en Pérez Latorre et al. (1993). Se menciona el dinamismo de cada una y las comunidades o grupos sintaxonómicos con las que entran en contacto catenal (geosigmetum).

\section{RESULTADOS}

Se han estudiado ocho series y subseries de vegetación climatófilas (mapa 1) que se reparten fundamentalmente en los pisos bioclimáticos termomediterráneo y mesomediterráneo.

En cuanto a composición y dinamismo es 


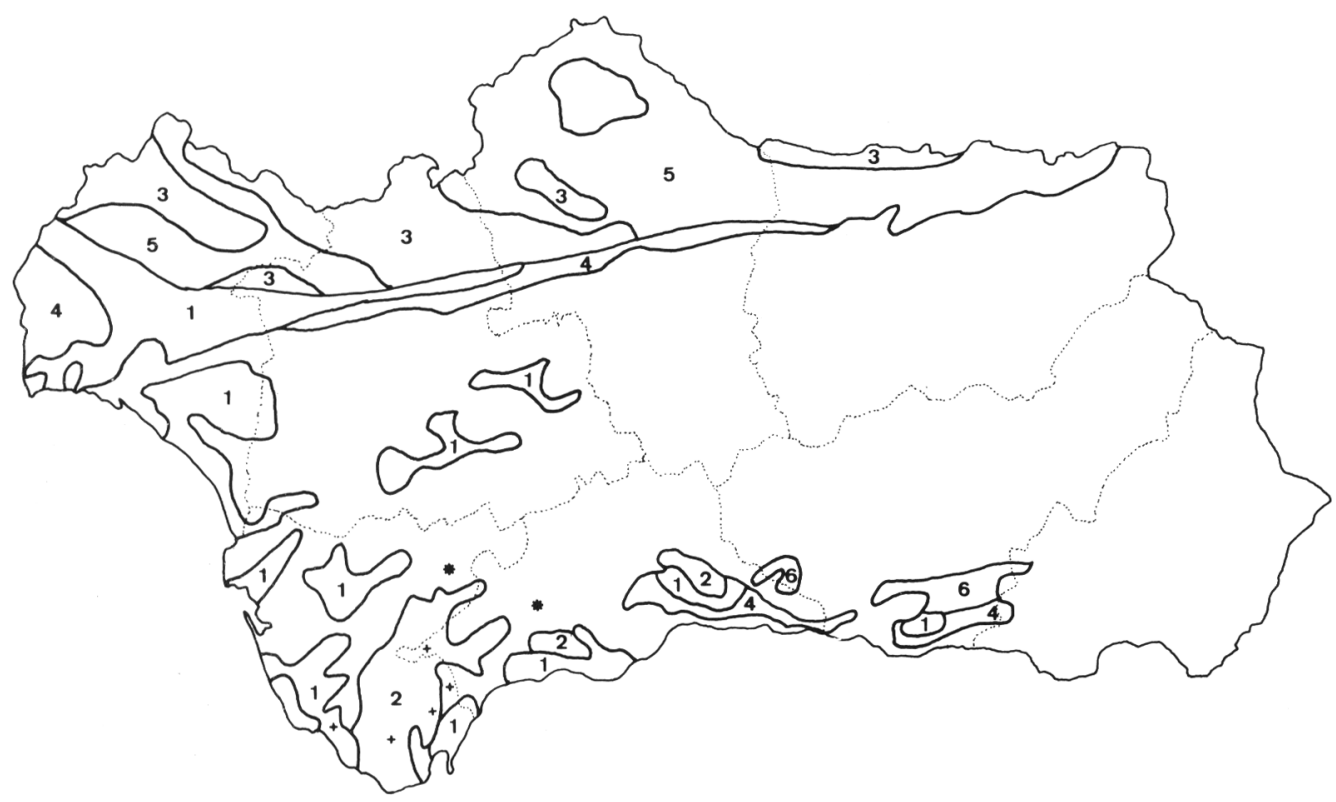

Mapa 1. Distribución en Andalucía de las series y subseries de vegetación caracterizadas por Quercus suber. Alcornocales: $1=$ Myrto communis-Querceto suberis $\mathrm{S}, 2=$ Teucrio baetici-Querceto suberis $\mathrm{S}, 3=$ Sanguisorbo hybridae-Querceto suberis S. Encinares con alcornoques: 4= Smilaci mauritanicae-Querceto rotundifoliae quercetoso suberis subsigmetum, 5= Pyro bourgaeanae-Querceto rotundifoliae quercetoso suberis subsigmetum, 6= Adenocarpo decorticantis-Querceto rotundifoliae quercetoso suberis subsigmetum, * = Paeonio coriaceae-Querceto rotundifoliae quercetoso fagineae subsigmetum variante con Quercus suber. Acebuchal con alcornoques: + = Tamo communis-Oleeto sylvestris quercetoso suberis subsigmetum. Distribution of the vegetation series with $Q$. suber in Andalusia, Spain. $Q$. suber woods $=1,2$ and 3. Q. rotundifolia \& $Q$. suber woods $=4,5,6$ and $*$. Olea sylvestris \& $Q$. suber woods $=+$.

notoria la diferencia existente entre las series constituidas por alcornocales en sentido estricto y los encinares o acebuchales con alcornoques. Los alcornocales presentan con frecuencia los herbazales esciohumícolas de Origanion virentis, madroñales de Ericion arboreae como vegetación preforestal y como etapa de matorral a brezales o brezal-jarales de Ericion umbellatae. Los encinares o acebuchales con alcornoques, sin embargo, sólo presentan los herbazales de Origanion virentis de modo fragmentario, llevando como etapa preforestal coscojares y lentiscares de Asparago-Rhamnion o Rhamno-Quercion y como etapa de matorral a jarales de UliciCistion ladaniferi.

\section{Piso bioclimático termomediterráneo.}

1.-Serie termomediterránea, Gaditano-OnuboAlgarviense, Bética, Luso-Extremadurense y norteafricana, subhúmeda y silicícolosabulícola del alcornoque (Quercus suber): Myrto communis-Querceto suberis sigmetum.

Se reconocen tres faciaciones condicionadas por la estructura del sustrato sobre el que se desarrollan y el sector biogeográfico en que están presentes.

La faciación típica, quercetoso suberis, se localiza sobre sustratos duros de naturaleza silícea en la provincia Bética y en el sector 
Aljíbico. El bosque, Myrto-Quercetum suberis, puede ser de alcornoques o mixto con quejigo (Quercus faginea). Por lo general presenta una cobertura no muy densa, salvo en umbrías y vaguadas en donde se desarrolla, algo empobrecido, el herbazal esciófilo de CalaminthoGallietum scabri. Este alcornocal es una formación en donde aparecen gran cantidad de arbustos termófilos (Calicotome villosa, Chamaerops humilis, Olea sylvestris y Pistacia lentiscus). La formación preforestal de este alcornocal es un madroñal de Cytiso-Arbutetum unedi quercetosum cocciferae el cual se transforma por degradación en un lentiscar de Asparago-Calicotometum villosae, con máxima representación en las margoareniscas aljíbicas, o en un coscojar de BupleuroPistacietum lentisci adenocarpetosum telonensis en las pizarras de los subsectores Alpujarreño, Malacitano y Axarquiense. La siguiente etapa de sustitución está constituída por jarales que en el subsector Aljíbico son de Calicotomo-Genistetum hirsutae genistetosum triacanthi, en el Marbellí de CalicotomoGenistetum hirsutae típico o genistetosum umbellatae en las zonas menos húmedas y de Lavandulo-Genistetum equisetiformis en el Malacitano-Axarquiense y AlpujarroGadorense. Sobre litosoles se desarrolla un pastizal oligótrofo de Tuberarion guttatae.

Generalmente, esta faciación contacta catenalmente con la vegetación ripícola termófila de saucedas, Equiseto-Salicetum pedicellatae y en las zonas más secas con adelfares de Rubo-Nerietum oleandri .

La faciación lavanduletoso luisieri aparece sobre sustratos duros en el sector Mariánico-Monchiquense. El bosque, MyrtoQuercetum suberis lavanduletosum luisieri, puede ser puro o mixto con quejigo (Quercus faginea) o encinas (Quercus rotundifolia). Por lo general presenta una cobertura no muy densa, salvo en umbrías y vaguadas. Presenta gran cantidad de arbustos termófilos en los claros (Chamaerops humilis, Olea sylvestris y
Pistacia lentiscus). Como orla sobre suelos profundos se desarrolla un madroñal de Phyllireo-Arbutetum unedi pistacietosum, que puede ser viburnetosum en orientaciones norte. En condiciones de alta cobertura arbórea y suelos con horizonte húmico bien desarrollado pueden aparecer fragmentos del herbazal esciófilo de Pimpinello-Origanetum virentis. La siguiente etapa de sustitución está constituída por jarales que en Sierra Morena occidental, subsector Araceno-Pacense, pertenecen al Ulici-Cistetum ladaniferi típico, el cual puede ser ericetosum australis en áreas más lluviosas y que se sustituye hacia el este por el Genisto-Cistetum ladaniferi típico o ericetosum australis en áreas de ombroclima superior. Sobre litosoles se desarrolla un pastizal oligótrofo de Tuberarion guttatae.

Generalmente, la faciación contacta catenalmente con comunidades ripícolas termófilas del Salicion salvifoliae (saucedas) y puntualmente con alisedas de ScrophularioAlnetum glutinosae.

La faciación halimietoso halimifolii se desarrolla sobre arenales de interior, rara vez costeros de los sectores Gaditano, Onubense, Aljíbico e Hispalense. Está caracterizada por la presencia de Halimium halimifolium, Halimium commutatum, Euphorbia baetica y otros fanerófitos psammófilos como Erica scoparia y Juniperus turbinata. El alcornocal, Myrto-Quercetum suberis halimietosum, es poco denso y con gran cantidad de arbustos, marcando la presencia de algunos geófitos en las zonas de alta humedad edáfica la variante de Arum italicum. Pueden aparecer representaciones puntuales de madroñales de CytisoArbutetum unedi quercetosum cocciferae en vaguadas húmedas del subsector Marbellí. Su primera etapa de sustitución está constituida por un lentiscar con sabinas de AsparagoRhamnetum oleoidis ericetosum scopariae en arenales sin hidromorfía acusada. En el sector Onubense (P.N. de Doñana), en arenales con hidromorfía cercana a la superficie, donde se 
desarrolla la variante higrófila de Arum italicum, puede aparecer un madroñal de Phyllireo-Arbutetum. unedi pistacietosum. El matorral de sustitución es diferente según las condiciones hídricas del sustrato. En el sector Onubense, sobre suelos secos, se desarrolla el jaguarzal de Halimio-Stauracanthetum genistoidis ulicetosum australis, mientras que en zonas donde la capa freática se acerca a la superficie se encuentra un brezal de EricoUlicetum australis. En los bordes de la marisma, con ombrotipo seco, el jaguarzal de sustitución es el Halimio-Cistetum libanotidis, mientras que en áreas de ombrotipo subhúmedo de las provincias de Cádiz, Sevilla y Málaga, aparecen jaguarzales con aulagas de CistoUlicetum australis. Como última etapa encontramos pastizales de hemicriptófitos psammófilos que pueden incluirse en Centaureo-Armerietum gaditanae, que llega fragmentariamente hasta los arenales marbellíes. El pastizal terofítico sobre suelos sueltos es de Anthyllido-Malcomion lacerae y en suelos consolidados de Tuberarion guttatae.

Hacia los arenales más secos, esta faciación contacta con las formaciones del sabinar sobre dunas estabilizadas de OsyrioJuniperetum turbinatae mientras que en arenales con fuerte hidromorfía, comunes en el sector Onubense, lo hace con las formaciones hidrofíticas de Ficario ranunculoidisFraxinetum angustifoliae.

2.-Serie termo-mesomediterránea, Aljíbica, Rondeña y Malacitano-Axarquiense, subhúmeda -húmeda- hiperhúmeda, silicícola del alcornoque (Quercus suber): Teucrio baetici-Querceto suberis sigmetum.

Alcornocal mesófilo con un área de distribución muy extensa y que presenta una gran variabilidad.

La faciación típica quercetoso suberis se localiza en las áreas de ombrotipo húmedo (hiperhúmedo), sobre areniscas o materiales esquistosos, en los pisos bioclimáticos termo y mesomediterráneo de los sectores Aljíbico y Rondeño. El bosque, Teucrio-Quercetum suberis, está dominado por el alcornoque y presenta una cobertura cercana al $100 \%$. El sotobosque es rico en criptófitos y hemicriptófitos nemorales esciohumícolas del herbazal de Calamintho-Gallietum scabri, el cual sobre las areniscas se enriquece en endemismos aljíbicos que permiten caracterizar la subasociación digitaletosum bocquetii. Dentro de esta faciación típica se reconocen tres variantes debidas a factores climáticos y biogeográficos. En algunas zonas altas del subsector Aljíbico, como la Sierra del Aljibe, Loma del Castillo, Sierras de Algeciras y parte superior del valle del río Genal, piso mesomediterráneo húmedo, se presenta la variante de Quercus pyrenaica. En el subsector Marbellí, el alcornocal se caracteriza por presentar al pinsapo como árbol acompañante, permitiendo distinguir la variante de Abies pinsapo. Una tercera variante es la de Pistacia lentiscus, que se desarrolla por todo el piso bioclimático termomediterráneo del sector Aljíbico. Las tres variantes llevan como etapa preforestal, en suelos profundos, un madroñal de CytisoArbutetum unedi caracterizado por Cytisus villosus, Genista monspessulana y Genista linifolia, madroñal que en las áreas más térmicas aparece como subasociación quercetosum cocciferae. Sobre suelos más acidificados, o en ambientes más abiertos del subsector Aljíbico, el madroñal se sustituye por un quejigal enano de Phyllireo-Quercetum lusitanicae que puede ser comunidad permanente en crestas y laderas muy rocosas, presentándose entonces como subasociación cytisetosum tribracteolati*, sobre todo en áreas cacuminales. Ambas formaciones (madroñal y quejigal enano), son sustituídos en suelos margoso-areniscosos más degradados y en zonas térmicas por el lentiscar con escobones de Asparago-Calicotometum villosae genistetosum 
linifoliae. Los matorrales de sustitución están constituídos por brezales en áreas de ombrotipo húmedo-hiperhúmedo y por jarales en las de ombrotipo subhúmedo-(húmedo). Los brezales desarrollados sobre areniscas y que provienen de la degradación de madroñales se incluyen en el Genisto tridentis-Stauracanthetum boivinii típico, apareciendo la subasociación quercetosum lusitanicae cuando el brezal proviene de degradación de quejigales enanos, y siendo ulicetosum baetici cuando se localizan sobre esquistos (areas más orientales del subsector Aljíbico) llegando esta última empobrecida al subsector Marbellí. En zonas de ombrotipo húmedo con incendios recurrentes se favorece el desarrollo del jaral de Calicotomo-Genistetum hirsutae genistetosum triacanthi, presentándose la subasociación típica de dicho jaral en las zonas de ombrotipo subhúmedo del subsector Marbellí. En el área de esta faciación, sobre litosoles muy lixiviados (taludes y cortafuegos) se desarrolla la original comunidad con atrapamoscas de StauracanthoDrosophylletum lusitanici. Sobre litosoles se desarrolla como etapa final de la faciación un pastizal oligótrofo de Tuberarion guttatae.

Catenalmente contacta en las zonas más elevadas con formaciones de Quercus pyrenaica y Quercus fruticosa posiblemente incluíbles en Arbuto-Quercetum pyrenaicae. Hacia los valles, el alcornocal contacta, en

(*)Phyllireo angustifoliae-Quercetum lusitanicae cytisetosum tribracteolati subass. nova. Typus: Cádiz, Algeciras, Sierra de Luna, Tajo de la Corza, 740 m., veg. pot. alcornocal con quejigo moruno, zona cacuminal, areniscas, mesomediterráneo hiperhúmedo. Especie característica y diferenciales de subasociación: Cytisus tribracteolatus 2, Cytisus grandiflorus 1, Stipa gigantea 1. Características de asociación y compañeras: Quercus lusitanica 3, Cistus populifolius major 3, Erica australis 1, Lavandula stoechas +, Teucrium baeticum 1, Smilax aspera + , Calluna vulgaris + , Arrenatherum bulbosum + , Digitalis bocqueti 1 , Tamus communis + , Ruscus aculeatus + . cauces anchos, con alisedas de Arisaro proboscidei-Alnetum glutinosae, mientras que en los arroyos que transcurren por barrancos umbrosos y profundos lo hace con ojaranzales de Frangulo-Rhododendretum baetici; pudiéndose encontrar también saucedas de EquisetoSalicetum pedicellatae y en zonas margosas, fresnedas de Ficario-Fraxinetum angustifoliae.

La faciación quercetoso canariensis aparece en áreas de tránsito del ombrotipo húmedo al hiperhúmedo, en vaguadas, zonas muy umbrías o con algún tipo de hidromorfía edáfica, siendo exclusiva del subsector Aljíbico. El bosque, Teucrio-Quercetum suberis quercetosum canariensis, está enriquecido respecto a la subasociación típica con quejigo moruno (Quercus canariensis, Quercus $x$ marianica) y helechos epífitos como Davallia canariensis. Le acompaña un herbazal esciófilo de Calamintho-Gallietum scabri lamietosum flexuosi. Lleva como primera etapa de sustitución en los suelos profundos un madroñal de Cytiso-Arbutetum unedi que en suelos muy húmedos aparece en su variante de Laurus nobilis, mientras que en suelos más degradados se encuentra un quejigal enano de Phyllireo-Quercetum lusitanicae típico, apareciendo en zonas rocosas y crestas como subasociación cytisetosum tribracteolati. Las siguientes etapas de sustitución son brezales de Genisto-Stauracanthetum boivinii quercetosum lusitanicae sobre areniscas y de Genisto-Stauracanthetum boivinii ulicetosum baetici sobre esquistos. En ambos sustratos aparece el brezal de atrapamoscas, StauracanthoDrosophylletum lusitanici, en taludes secos y cortafuegos. La última etapa de esta faciación es un pastizal terofítico de Tuberarion guttatae.

Catenalmente y hacia el fondo de valles y en umbrías la faciación contacta con los quejigares morunos de Rusco hypophilliQuercetum canariensis. Ya en contacto directo con el agua de los arroyos umbrosos contacta con ojaranzales de Frangulo-Rhododendretum baetici 0 , en cauces abiertos, con alisedas de 
Arisaro-Alnetum glutinosae.

La faciación quercetoso rotundifoliae ocupa áreas mesomediterráneas de ombrotipo subhúmedo-húmedo sobre suelos poco profundos $\mathrm{y} / \mathrm{o}$ con introgresiones de materiales básicos fundamentalmente en los subsectores Axarquiense y Aljíbico. El alcornocal, TeucrioQuercetum suberis quercetosum rotundifoliae, es un bosque mixto de alcornoque, encina y quejigo, rico en arbustos de Ericion arboreae y en genisteas retamoides. En zonas conservadas aparece el herbazal esciohumícola de Calamintho-Gallietum scabri algo empobrecido en táxones característicos pero con Doronicum plantagineum y Centaurea sempervirens. La primera etapa de sustitución en el subsector Aljíbico es un madroñal de Cytiso-Arbutetum unedi típico, mientras que en el Axarquiense el madroñal se presenta como la subasociación bupleuretosum fruticosi, caracterizada por la ausencia de plantas termófilas y la presencia de Lonicera implexa y Bupleurum fruticosum entre otras. La siguiente etapa de sustitución es un brezaljaral de Genisto-Stauracanthetum boivinii ulicetosum baetici en el Aljíbico y un jaral de Lavandulo-Genistetum equisetiformis con Cistus ladanifer en el Axarquiense. La etapa final de esta faciación es un pastizal terofítico deTuberarion guttatae.

Estos alcornocales pueden entrar en contacto catenal con encinares de PaeonioQuercetum rotundifoliae ya sobre suelos calizos o con saucedas de Equiseto-Salicetum pedicellatae y choperas de Rubio-Populetum albae hacia los cauces fluviales.

3.-Serie termomediterránea, Bética, MariánicoMonchiquense y Rifeña, seco-subhúmeda e indiferente edáfica de la encina carrasca (Quercus rotundifolia): Smilaci mauritanicaeQuerceto rotundifoliae, subserie silicícola, seco superior - subhúmeda inferior con alcornoque (Quercus suber) quercetoso suberis sigmetum.
Estos encinares con alcornoques se localizan en aquellas zonas de transición entre el ombrotipo seco y el subhúmedo. El bosque, Smilaci-Quercetum rotundifoliae quercetosum suberis, presenta cobertura variable dependiendo de las condiciones hídricas y del uso del territorio, apareciendo como variante de Pyrus bourgeana en el sector MariánicoMonchiquense. La primera etapa de sustitución, en el sector Mariánico-Monchiquense y enclaves silíceos del sector Hispalense, la constituyen coscojares de Asparago-Rhamnetum oleoidis, mientras que en los sectores Malacitano-Axarquiense y AlpujarroGadorense aparecen lentiscares de BupleuroPistacietum lentisci adenocarpetosum telonensis sustituído en taludes umbrosos, laderas de barrancos y suelos conservados por formaciones arbustivas de BupleuroOnonidetum speciosae. El matorral de sustitución en el Malacitano-Axarquiense y Alpujarro-Gadorense es un jaral de LavanduloGenistetum equisetiformis con Cistus ladanifer, o conCalicotome intermedia en áreas costeras, y en el Mariánico-Monchiquense y las rañas del valle del Guadalquivir (Hispalense) un jaral de Genisto-Cistetum ladaniferi. Por último, sobre litosoles, aparece el pastizal terofítico de Tuberarion guttatae.

Hacia los cauces, generalmente estacionales, la faciación contacta catenalmente con adelfares de Rubo-Nerietum oleandri y en el Mariánico-Monchiquense también con tamujares de Pyro-Securinegetum tinctoriae.

4.-Serie termomediterránea, Tingitana, Gaditana, Aljíbica y Bética, subhúmedo-húmeda, verticícola del acebuche (Olea sylvestris): Tamo communis-Oleeto sylvestris, subserie húmeda sobre margoareniscas con alcornoque (Quercus suber) quercetoso suberis subsigmetum. 
Acebuchal con alcornoques que se desarrolla en Andalucía en los sectores Gaditano y Aljíbico, sobre margoareniscas, generalmente en relieves poco acentuados. El bosque, TamoOleetum sylvestris quercetosum suberis, presenta el aspecto de una formación arbustiva densa dominada por Olea sylvestris y Pistacia lentiscus entre los que sobresalen alcornoques dispersos. La humanización del territorio hace que el bosque se transforme en el lentiscar con escobones de Asparago-Calicotometum villosae genistetosum linifoliae. La proliferación de incendios y rozas periódicas hace que el lentiscar se degrade a un aulagar incluíble en Calicotomo-Genistetum hirsutae genistetosum triacanthi donde predominan Ulex borgiae, Genista triacanthos, Cistus monspeliensis, Phlomis purpurea, etc. Como última etapa de sustitución aparece un pastizal anual de Tuberarion guttatae enriquecido en especies de Poetea bulbosae debido a la presencia habitual de ganado.

En suelos margosos algo hidromorfos la faciación contacta con fresnedas de FicarioFraxinetum angustifoliae.

\section{Piso bioclimático mesomediterráneo.}

5.-Serie mesomediterránea, LusoExtremadurense, subhúmedo-húmeda, silicícola del alcornoque (Quercus suber): Sanguisorbo hybridae-Querceto suberis sigmetum.

En Andalucía está representada en el sector Mariánico-Monchiquese y reconocemos tres faciaciones de esta serie diferenciables fundamentalmente en base a sus requerimientos hídricos y edáficos.

La faciación típica, quercetoso suberis, ocupa las áreas menos lluviosas del sector. El bosque, Sanguisorbo-Quercetum suberis, es una formación de alcornoques que pueden acompañarse de quejigos y encinas. En um- brías y vaguadas, donde el suelo conserva el horizonte húmico, se desarrolla el herbazal esciófilo de Pimpinello-Origanetum virentis. Como orla preforestal se desarrolla un madroñal de Phyllireo-Arbutetum unedi, apareciendo en vaguadas y umbrías la subasociación viburnetosum tini que puede presentarse en el subsector Araceno-Pacense bajo dos variantes, con Genista linifolia (centro de la Sierra Norte sevillana) y con Genista falcata (Sierra de Aracena, Huelva). La siguiente etapa de sustitución la constituyen brezales y jarales dependiendo fundamentalmente del ombroclima. En el subsector Araceno-Pacense sobre suelos poco profundos (entisoles) con ombrotipo húmedo-subhúmedo superior, se desarrolla un brezal de UliciEricetum umbellatae, mientras que sobre suelos aún algo conservados y húmedos aparece un jaral-brezal de Erico-Cistetum populifolii que puede ser halimietosum viscosii en zonas mas frías. En el subsector Marianense con ombroclima subhúmedo superior se localiza un brezal-jaral de Polygalo-Cistetum populifolii. Los jarales aparecen generalmente en áreas de ombrotipo subhúmedo, siendo en la mitad occidental del subsector AracenoPacense de Ulici-Cistetum ladaniferi ericetosum australis mientras que en su mitad oriental y en el subsector Marianense el jaral es de Genisto-Cistetum ladaniferi ericetosum australis. Por último, cierra la dinámica de la faciación el pastizal silicícola de Tuberarion guttatae.

La faciación contacta catenalmente con vegetación ripícola de alisedas de Scrophulario scorodoniae-Alnetum glutinosae, saucedas de Salicion salvifoliae y fresnedas y olmedas de Populion albae.

Dentro de esta faciación tenemos que comentar la existencia, sobre calizas y con ombrotipo húmedo, en la Sierra de Aracena y puntualmente en la Sierra Norte de Sevilla, de un alcornocal que se diferencia de la faciación 
típica en las etapas de matorral y pastizal terofítico. Dicho matorral corresponde a un jaral de Ulici eriocladi-Cistetum ladaniferi cistetosum albidi*, jaral con especies de Rosmarinetea debido a la influencia de la roca madre calcárea, la cual influye también en el pastizal terofítico, constituido por combinación de especies de Tuberarion guttatae y Brachypodion dystachiae.

Esta variante calcícola contacta catenalmente con la faciación típica del alcornocal sobre suelos ya silíceos y con coscojares de Rhamno-Quercion cocciferae hacia los afloramientos calizos cumbreños.

La faciación quercetoso canariensis se desarrolla en áreas de ombrotipo húmedo y con aporte extra de humedad por compensación hídrica de origen topográfico (barrancos profundos, laderas umbrías, etc.), localizada hasta ahora únicamente en el subsector Araceno-Pacense (Sierra de Aracena). Acompañando al alcornoque aparecen quejigos (Quercus canariensis, Quercus $x$ marianica, Quercus faginea broteroi) y algunos táxones higrófilos endémicos como Campanula primulifolia. En este ambiente de quejigal es frecuente el herbazal esciohumícola del Pimpinello-Origanetum virentis. Como etapa preforestal, en suelos bien conservados, aparece un madroñal de Phyllireo-Arbutetum unedi viburnetosum, que se transforma en un jaral-

(*)Ulici eriocladi-Cistetum ladaniferi cistetosum albidi subass. nova. Typus: Huelva, Linares de la Sierra, Sierra de San Ginés, 755 m., veg. pot. alcornocal, calizas paleozoicas, mesomediterráneo húmedo. Especies diferenciales de subasociación: Cistus albidus 3, Hipocrepis scabra 3, Satureja graeca +. Características de asociación, clase y compañeras: Ulex eriocladus + , Phlomis purpurea 1, Genista hirsuta + , Lavandula luisieri + , Cistus salvifolius 2, Bupleurum fruticosum + , Teucrium haenseleri + , Rosmarinus officinalis +, Elaeoselinum phoetidum. + , Ruta montana 1, Cistus crispus + , Astragalus lusitanicus + , Thapsia maxima + , Helichrysum stoechas + . brezal de Erico-Cistetum populifolii si el suelo se hace menos profundo pero se mantiene un cierto grado de humedad. La última etapa la constituye un pastizal anual de Tuberarion guttatae.

Contacta en fondo de valles con alisedas de Scrophulario-Alnetum glutinosae o saucedas de Salicion salvifoliae.

La faciación quercetoso pyrenaicae se desarrolla en áreas de ombrotipo húmedosubhúmedo superior, en el piso bioclimático mesomediterráneo medio-inferior, encontrándose de modo puntual por todo el sector Mariánico-Monchiquense. El alcornocal toma el aspecto de un bosque mixto de robles (Quercus pyrenaica) alcornoque y quejigo, siendo frecuente su sustitución por antiguas plantaciones de castaños. Presenta un sotobosque rico en hemicriptófitos que conforman un herbazal de Pimpinello-Origanetum virentis, caracterizado en la sierra de Aracena por Centaurea tartesiana. Cuando Quercus pyrenaica comienza a dominar estas formaciones (aumento de precipitaciones y altitud) aparecen piornales de Cytisus striatus y Genista falcata (sierra de Aracena) o Genista tournefortii (Sierra Quintana). Aparece acompañando al estrato arbóreo sobre suelos profundos un madroñal de Phyllireo-Arbutetum unedi viburnetosum que, en el Araceno-Pacense, lleva como diferencial Genista falcata y en el Marianense Juniperus badia. En zonas puntuales de la Sierra de Aracena, sobre suelos menos profundos, aparece un nanobosquete de robledilla de Phyllireo-Quercetum lusitanicae quercetosum rotundifoliae. El brezal de sustitución en el Araceno-Pacense es de UliciEricetum umbellatae en suelos degradados o de Erico-Cistetum populifolii en suelos más húmedos y conservados. El brezal-jaral que aparece sobre suelos algo degradados del subsector Marianense es de Polygalo-Cistetum populifolii que se transforma, sobre suelos muy degradados, en nanobrezales de HalimioEricetum umbellatae. La etapa final es un 
pastizal terofítico de Tuberarion guttatae.

Hacia zonas más elevadas o más umbrías y húmedas, contacta catenalmente con melojares de Arbuto-Quercetum pyrenaicae, y en los cauces con alisedas de ScrophularioAlnetum glutinosae.

6.-Serie mesomediterránea, LusoExtremadurense, seco-subhúmeda, silicícola de la encina (Quercus rotundifolia): Pyro bourgaeanae-Querceto rotundifoliae, subserie subhúmeda y termófila con alcornoque (Quercus suber) quercetoso suberis subsigmetum.

Esta faciación se desarrolla en zonas serranas, mesomediterráneas, térmicas, del sector Mariánico-Monchiquense con ombrotipo subhúmedo. El bosque, Pyro-Quercetum rotundifoliae quercetosum suberis, es una formación mixta de encinas, coscojas, alcornoques y a veces quejigos. En umbrías o zonas bien conservadas, aparecen herbazales vivaces de Pimpinello-Origanetum virentis algo empobrecidos. Sobre suelos un poco degradados se instala un coscojar de HyacinthoidoQuercetum cocciferae, muy denso y de gran cobertura. Por el contrario, en vaguadas y umbrías con suelos bién conservados, pueden encontrarse madroñales de PhyllireoArbutetum unedi, empobrecidos en especies de Ericion arboreae y con presencia de táxones de Rhamno-Quercion. En el subsector Araceno-Pacense, la siguiente etapa dinámica es un jaral de Ulici-Cistetum ladaniferi típico, que puede ser ericetosum australis en zonas más lluviosas. En el subsector Marianense y zonas más nororientales del Araceno-Pacense aparece un jaral de Genisto-Cistetum ladaniferi, que también puede ser ericetosum australis (más humedad) o halimietosum viscosii (más continentalidad). En taludes y crestas pizarrosas del subsector Araceno-Pacense aparecen formaciones de Ulici-Cistetum ladaniferi ericetosum australis con Genista polyanthos. Como etapa final aparece un pastizal anual de Tuberarion guttatae.

Contacta catenalmente con vegetación higrófila de saucedas de Salicion salvifoliae, fresnedas de Populion albae o alisedas de Scrophulario-Alnetum glutinosae. En cauces mas estacionales contacta con tamujares de Pyro-Securinegetum tinctoreae y en areas mineras del Andévalo (Huelva) con brezales de Junco-Ericetum andevalensis ulicetosum eriocladi.

7.-Serie mesomediterránea; Bética, secosubhúmeda-húmeda basófila de la encina (Quercus rotundifolia): Paeonio coriaceaeQuerceto rotundifoliae subserie húmeda con quejigo (Quercus faginea) quercetoso fagineae subsigmetum, variante sobre suelos descarbonatados con Quercus suber.

Variante muy localizada en el sector Rondeño, Nava de San Luis (Parauta, Málaga) y Cerro del Montón (Grazalema, Cádiz) caracterizada por desarrollarse sobre suelos calizos descarbonatados, ombrotipo húmedo(hiperhúmedo) y piso bioclimático mesomediterráneo. El bosque es mixto, con encinas, alcornoques, pinsapos y quejigos, apareciendo táxones silicícolas como Cytisus grandiflorus, Erica arborea, etc. El pastizal nemoral está influenciado por la cercanía geográfica de los alcornocales de Teucrio-Querceto suberis sigmetum siendo incluible en un empobrecido Calamintho-Gallietum scabri. Su primera etapa de sustitución la constituye un coscojar de Crataego-Quercetum cocciferae variante de Cytisus grandiflorus, caracterizado por táxones silicícolas como Erica scoparia, Erica arborea, Cytisus grandiflorus y Arbutus unedo. El jaral, muy fragmentario, es incluible en el Cistion laurifolii y está caracterizado por Cistus salvifolius, Cistus laurifolius, Ulex baeticus, Erica australis y Lavandula stoechas. 
El pastizal terofítico presenta mezcla de especies de Tuberarion guttatae y Brachypodietalia al aflorar la roca madre calcárea.

Contacta con los encinares del PaeonioQuercetum rotundifoliae quercetosum fagineae o pinsapares de Paeonio-Abietetum pinsapo en areas calizas con suelos carbonatados.

8.-Serie supra-mesomediterránea, Bética, secosubhúmeda, silicícola de la encina (Quercus rotundifolia): Adenocarpo decorticantisQuerceto rotundifoliae, subserie mesomediterránea subhúmeda con alcornoque (Quercus suber) quercetoso suberis subsigmetum.

Esta subserie se desarrolla en los sectores Almijaro-Granatense, Alpujarro-Gadorense y Nevadense ocupando una estrecha banda bioclimática altitudinal. El bosque, Adenocarpo-Quercetum rotundifoliae quercetosum suberis, es una formación mixta de encinas, alcornoques y quejigos (en zonas mas húmedas). En determinadas localidades y debido al uso del territorio, el alcornoque ha sido favorecido haciéndose dominante (Haza del Lino, Granada) por lo que estas formaciones han sido consideradas por algunos autores como alcornocales, sin embargo la presencia de Adenocarpus decorticans y Quercus rotundifolia nos indica la potencialidad de un encinar supra-mesomediterráneo con alcornoques. La primera etapa de sustitución es un piornal de Cytiso-Adenocarpetum decorticantis cytisetosum grandiflorii enriquecida con plantas de los espinares caducifolios como Crataegus monogyna y Rosa sp., mas termófila que la subasociación típica. En suelos degradados aparece un jaral de LavanduloGenistetum equisetiformis cistetosum laurifolii. Es de destacar, en las zonas más frías y elevadas, la presencia de lastonares de Helictotricho-Festucetum scariosae. Los pastizales anuales se incluyen enTuberarion guttatae.

Contacta catenalmente, en el supramediterráneo subhúmedo, con melojares del Adenocarpo decorticantis-Quercetum pyrenaicae y en el mesomediterráneo seco con encinares de AdenocarpoQuercetum rotundifoliae. En los cauces se pone en contacto con saucedas de Salicion salvifoliae y choperas y olmedas de Populion albae.

\section{Dinamismo de las series}

A continuación se esquematiza la composición de cada subserie de vegetación, citando las asociaciones de que se compone ordenadas según el dinamismo sucesional desde bosque a pastizal. Los símbolos empleados son: \#=etapa boscosa y pastizales esciohumícolas, ${ }^{*}=$ formaciones preforestales y de orla, ${ }^{* *}=$ jarales y brezales, +=pastizales.

1a. Myrto-Querceto suberis quercetoso suberis subsigmetum

\# Myrto-Quercetum suberis/Calamintho-Gallietum scabri

* Cytiso-Arbutetum unedi quercetosum cocciferael Asparago-Calicotometum villosae/BupleuroPistacietum lentisci adenocarpetosum telonensis

* Calicotomo-Genistetum hirsutae (típico, genistetosum triacanthi, genistetosum umbellatae)/ Lavandulo-Genistetum equisetiformis var. Cistus ladanifer

+ Tuberarion guttatae

1b. Myrto-Querceto suberis lavanduletoso luisieri subsigmetum

\# Myrto-Quercetum suberis lavanduletosum luisieri/Pimpinello-Origanetum virentis

* Phyllireo-Arbutetum unedi (viburnetosum, pistacietosum)

** Ulici-Cistetum ladaniferi (típico, ericetosum australis)/Genisto-Cistetum ladaniferi (típico, ericetosum australis)

+ Tuberarion guttatae 


\section{1c. Myrto-Querceto suberis halimietoso halimifolii} subsigmetum

\# Myrto-Quercetum suberis halimietosum halimifolii (var. típica, var. de Arum italicum)

* Asparago-Rhamnetum oleoidis ericetosum scopariae/Phyllireo-Arbutetum unedi pistacietosum lentisci/Cytiso-Arbutetum unedi quercetosum cocciferae

** Erico-Ulicetum australis/Halimio-Stauracanthetum genistoidis ulicetosum australis/Halimio-Cistetum libanotidis/Cisto-Ulicetum australis/CentaureoArmerietum gaditanae

+ Anthyllido-Malcomion lacerae/Tuberarion guttatae

\section{2a. Teucrio-Querceto suberis quercetoso suberis} subsigmetum

\# Teucrio-Quercetum suberis (var. Quercus pyrenaica, var. Abies pinsapo, var. Pistacia lentiscus)/Calamintho-Gallietum scabri (típico, digitaletosum bocquetti)

* Cytiso-Arbutetum unedi (típico, quercetosum cocciferae)/Phyllireo-Quercetum lusitanicae (típico, cytisetosum tribracteolati)/AsparagoCalicotometum villosae genistetosum linifoliae

** Calicotomo-Genistetum hirsutae (típico, genistetosum triacanthi)/Genisto-Stauracanthetum boivinii (típico, quercetosum lusitanicae, ulicetosum baetici)/Stauracantho-Drosophylletum lusitanici

+ Tuberarion guttatae

2b. Teucrio-Querceto suberis quercetoso canariensis subsigmetum

\# Teucrio-Quercetum suberis quercetosum canariensis/ Calamintho-Gallietum scabri lamietosum flexuosi

* Phyllireo-Quercetum lusitanicae (típico, cytisetosum tribracteolati)/Cytiso-Arbutetum unedi var. Laurus nobilis

* Genisto-Stauracanthetum boivinii (típico/ quercetosum lusitanicae/ulicetosum baetici)/ Stauracantho-Drosophylletum lusitanici

+ Tuberarion guttatae

2c. Teucrio-Querceto suberis quercetoso rotundifoliae subsigmetum

\# Teucrio-Quercetum suberis quercetosum rotundifoliae/Calamintho-Gallietum scabri

* Cytiso-Arbutetum unedi (típico, bupleuretosum fruticosi)

** Genisto-Stauracanthetum boivinii ulicetosum baetici/Lavandulo-Genistetum equisetiformis (var. Cistus ladanifer).

+ Tuberarion guttatae

\section{Smilaci-Querceto rotundifoliae quercetoso} suberis subsigmetum

\# Smilaci-Quercetum rotundifoliae quercetosum suberis (var. típica, var. Pyrus bourgaeana)

* Asparago-Rhamnetum oleoidis/Bupleuro-Pistacietum adenocarpetosum/Bupleuro-Ononidetum speciosae

** Genisto-Cistetum ladaniferi/Lavandulo-Genistetum equisetiformis (var. C.ladanifer, var. Calicotome intermedia) + Tuberarion guttatae

4. Tamo-Oleeto sylvestris quercetoso suberis subsigmetum

\# Tamo-Oleetum sylvestris quercetosum suberis

* Asparago-Calicotometum villosae genistetosum linifoliae

** Calicotomo-Genistetum hirsutae genistetosum triacanthi

+ Tuberarion guttatae/Poetea bulbosae

5a. Sanguisorbo-Querceto suberis quercetoso suberis subsigmetum

\# Sanguisorbo-Quercetum suberis/PimpinelloOriganetum virentis

* Phyllireo-Arbutetum unedi (típico, viburnetosum tini)

** Ulici-Ericetum umbellatae/Erico-Cistetum populifolii (típico, halimietosum viscosii)/PolygaloCistetum populifolii/Ulici-Cistetum ladaniferi ericetosum australis/Genisto-Cistetum ladaniferi ericetosum australis

+ Tuberarion guttatae

5b. Sanguisorbo-Querceto suberis quercetoso canariensis subsigmetum

\# Sanguisorbo-Quercetum suberis quercetosum canariensis/Pimpinello-Origanetum virentis

* Phyllireo-Arbutetum unedi viburnetosum tini

** Erico-Cistetum populifolii

+ Tuberarion guttatae

5c. Sanguisorbo-Querceto suberis quercetoso pyrenaicae subsigmetum

\# Sanguisorbo-Quercetum suberis quercetosum 
pyrenaicae/Pimpinello-Origanetum virentis

* Phyllireo-Arbutetum unedi viburnetosum tini (var. Genista falcata, var. Genista linifolia)/PhyllireoQuercetum lusitanicae quercetosum rotundifoliae ** Erico-Cistetum populifolii/Polygalo-Cistetum populifolii/Ulici-Ericetum umbellatae/HalimioEricetum umbellatae

+ Tuberarion guttatae

5d. Sanguisorbo-Querceto suberis quercetoso suberis subsigmetum var. calcícola

\# Sanguisorbo-Quercetum suberis/PimpinelloOriganetum virentis

* Phyllireo-Arbutetum unedi viburnetosum tini (var. Genista falcata, var. Genista linifolia)

** Ulici-Cistetum ladaniferi cistetosum albidi

+ Tuberarion guttatae/Brachypodion dystachiae

\section{Pyro-Querceto rotundifoliae quercetoso suberis subsigmetum \\ \# Pyro-Quercetum rotundifoliae quercetosum suberis/Pimpinello-Origanetum virentis \\ * Hyacinthoido-Quercetum cocciferae/Phyllireo- Arbutetum unedi \\ ** Genisto-Cistetum ladaniferi (típico, ericetosum australis, halimietosum viscosii)/Uilici-Cistetum ladaniferi (típico, ericetosum australis) \\ + Tuberarion guttatae}

\section{Paeonio-Querceto rotundifoliae quercetoso} fagineae subsigmetum var. de Quercus suber \# Paeonio-Quercetum rotundifoliae quercetosum fagineae var. de Quercus suber/CalaminthoGallietum scabri

* Crataego-Quercetum cocciferae var. de Cytisus grandiflorus

** Cistion laurifolii

+ Tuberarion guttatae/Brachypodion dystachiae

\section{Adenocarpo-Querceto rotundifoliae quercetoso} suberis subsigmetum

\# Adenocarpo-Quercetum rotundifoliae quercetosum suberis

* Cytiso-Adenocarpetum decorticantis cytisetosum grandiflorii

** Lavandulo-Genistetum equisetiformis cistetosum laurifolii/Helictotricho-Festucetum scariosae + Tuberarion guttatae
AGRADECIMIENTOS. Este trabajo se ha realizado con la ayuda concedida por la Consejería de Educación y Ciencia de la Junta de Andalucía al Grupo de Investigación 4013.

\section{BIBLIOGRAFÍA}

ACHHAL, A., O. AKABLI, M. BARBERO; A. BENABID; A. M'HIRIT; C. PEYRE, P. QUEZEL \& S. RIVAS-MARTÍNEZ - 1979- A propos de la valeur bioclimatique et dynamique de quelques essences forestières au Maroc. Ecologia Mediterranea, 5: 211-249.

ASENSI, A. y B. DÍEZ -1987- Andalucía Occidental. En: La vegetación de España. Peinado Lorca y S. Rivas Martínez (eds.).

BARBERO, M., P. QUÉZEL y S. RIVASMARTÍNEZ - 1981- Contribution à l'etude des groupements forestiers et preforestiers du Maroc. Phytocoenologia, 9(3): 311-412.

BELLOT RODRÍGUEZ, F. -1945- La asociación del Quercus suber L. en el Quercion ilicis de la Mariánica y Oretana. Boletim da Sociedade Broteriana, 19(2): 539-564.

BENABID, A. -1984- Etude phytoecologique des pleupements forestiers et preforestiers du Rif centro-occidental (Maroc). Travaux de l'Institut Scientifique. Série Botanique, 34. Rabat.

BENABID, A. \& FENNANE, M. -1994. Connaissances sur la végétation du Maroc: Phytogéographie, Phytosociologie et séries de végétation. Lazaroa, 14: 21-97.

CANO, E. y F. VALLE -1989- Las series de vegetación de Sierra Morena Oriental. Bol. Inst. Estudios Giennenses, 137: 75-87.

CEBALLOS, L. y M. MARTÍN BOLAÑOS -1930Estudio sobre la vegetación forestal de la provincia de Cádiz. Ingenieros de Montes del I.F.I.E. Madrid.

CEBALLOS, L. y C. VICIOSO - 1933-Estudio sobre la vegetación y la flora forestal de la provincia de Málaga. IX Inspección del I.C.O.N.A. Madrid.

DE LEÓN LLAMAZARES, A. -1989a- Caracterización agroclimática de la provincia de Almería. M. A. P. A.

DE LEÓN LLAMAZARES, A. -1989b- Caracterización agroclimática de la provincia de Cádiz. M. A. P. A.

DE LEÓN LLAMAZARES, A. -1989c-Caracteri- 
zación agroclimática de la provincia de Córdoba. M. A. P. A.

DE LEÓN LLAMAZARES, A. -1989d- Caracterización agroclimática de la provincia de Granada. M. A. P. A.

DE LEÓN LLAMAZARES, A. -1989e- Caracterización agroclimática de la provincia de Huelva. M. A. P. A.

DE LEÓN LLAMAZARES, A. -1989f- Caracterización agroclimática de la provincia de Jaén. M. A. P. A.

DE LEÓN LLAMAZARES, A. -1989g- Caracterización agroclimática de la provincia de Málaga. M. A. P. A.

DE LEÓN LLAMAZARES, A. -1989h- Caracterización agroclimática de la provincia de Sevilla. M. A. P. A.

DÍEZ, B., J. CUENCA y A. ASENSI -1988- Datos sobre la vegetación del subsector Aljíbico (provincia Gaditano-Onubo-Algaviense). Lazaroa, 9: 315-332.

GEHÙ, J.M. \& S. RIVAS-MARTÍNEZ -1981Notions fondamentales de Phytosociologie. Berichte der Internationalen Vereiningung fur Vegetationskunde, Syntaxonomie, págs. 5-53.

LADERO, M., F. NAVARRO, C. J. VALLE, J. L. PÉREZ CHISCANO, M. T. SANTOS BOBILLO, T. RUÍZ TÉllEZ, M. I. FERNÁNDEZ ARIAS, A. VALDÉS FRANZI y F. J. GONZÁLEZ -1985- Comunidades herbáceas de lindero en los bosques Carpetano-Ibérico-Leoneses y Luso-Extremadurenses. Studia Botánica, 4: 7-26.

LADERO, M. -1987- España Lusoextremadurense. En: La vegetación de España. Peinado Lorca y S. Rivas Martínez (eds.).

LADERO, M., J. L. PÉREZ CHISCANO, M. T. SANTOS, C. J. VALLE y A. AMOR -1990Encinares Luso-Extremadurenses y sus etapas pre-climácicas. Acta Bot. Malacitana, 15: 323329.

MARTÍNEZ PARRAS, J. M. y M. PEINADO LORCA -1987a- Andalucía Oriental. En: La vegetación de España. Peinado Lorca, M. y S. Rivas Martínez (eds.).

MARTÍNEZ PARRAS, J. M., M. PEINADO y M.
DE LA CRUZ -1987b- Aportación al estudio fitosociológico de los matorrales del Sector Rondeño. Studia Botanica, 6: 39-45.

NIETO CALDERA, J. M., A. V. PÉREZ LATORRE y B. CABEZUDO -1990- Datos sobre la vegetación silicícola de Andalucía I. Acta Bot. Malacitana, 15: 179-192.

PÉREZ CHISCANO, J. L. -1976- Charnecales y Madroñales de la Provincia de Badajoz. Anales Jard.. Bot. Madrid, 33: 215-238.

PÉREZ LATORRE, A.V., J. M. NIETO CALDERA y B. CABEZUDO - 1993- Contribución al conocimiento de la vegetación de Andalucía. II. Los alcornocales. Acta Bot. Malacitana, 18: 223258.

PONS, A. \& M. THINON -1987- The role of fire from palaeoecological data. Ecologia Mediterranea, 13(4): 3-11.

PRIETO, P. y P. ESPINOSA -1975- El alcornocal del Haza del Lino. Trab. Dep. Bot. Univ. Granada, 3 (1): 45-59.

QUÉZEL, P., M. BARBERO; A. BENABID; M. LOISEL y S. RIVAS-MARTÍNEZ -1988Contribution a l'etude des groupements préforestiers et des matorrals rifains. Ecologia Mediterranea, 14(1): 77-122.

RIVAS GODAY, S. -1954- Aportaciones a la fitosociología hispánica. Anales Jard.. Bot. Madrid, 13: 335-423.

RIVAS GODAY, S., E. FERNÁNDEZ GALIANO y S. RIVAS-MARTÍNEZ -1962- Vegetación Natural. En: Estudio Agrobiológico de la provincia de Sevilla. I. N. I. A. Sevilla.

RIVAS GODAY, S. -1964b- Vegetación y flórula de la cuenca extremeña del Guadiana. Serv. Publ. Excma. Dip. Prov. Badajoz.

RIVAS GODAY, S., E. FERNÁNDEZ GALIANO y S. RIVAS-MARTÍNEZ -1965- Vegetación natural. En: Estudio agrobiológico de la provincia de Cádiz. I. N. I. A. Sevilla.

RIVAS GODAY, S. y S. RIVAS-MARTÍNEZ 1971 a- Vegetación natural. En: Estudio agrobiológico de la provincia de Córdoba. I. N. I. A. Sevilla.

RIVAS GODAY, S. y S. RIVAS-MARTÍNEZ - 
1971b-Vegetación potencial de la provincia de Granada. Trab. Dep. Botánica y Fis. Veg., 4: 385. Univ. Complutense. Madrid.

RIVAS-MARTÍNEZ, S. -1974- La vegetación de la clase Quercetea ilicis en España y Portugal. Anales Jard.. Bot. Madrid, 31: 205-259.

RIVAS-MARTÍNEZ, S. -1976- Sinfitosociología, una nueva metodología para el estudio del Paisaje Vegetal. Anales Jard.. Bot. Madrid, 33: 179-188.

RIVAS-MARTÍNEZ, S. -1979- Brezales y jarales de Europa Occidental (Revisión de las clases Calluno-Ulicetea y Cisto-Lavanduletea). Lazaroa, 1: 5-128.

RIVAS-MARTÍNEZ, S., M. COSTA, S. CASTROVIEJO y E. VALDÉS -1980- Vegetación de Doñana. Lazaroā, 2: 5-189.

RIVAS-MARTÍNEZ, S. -1985-Biogeografía y Vegetación. Real Academia de Ciencias Exactas, Físicas y Naturales. Madrid.
RIVAS-MARTÍNEZ, S. -1987- Memoria y Mapas de las Series de Vegetación de España. M. A. P. A.-I. C. O. N. A.

RIVAS-MARTÍNEZ, S., M. LOUSA, T. E. DÍAZ, F. FERNÁNDEZ GONZALEZ y J. C. COSTA 1990- La vegetación del sur de Portugal (Sado, Alentejo y Algarve). Itinera Geobotanica, 3: 5126.

Aceptado para su publicación en Junio de 1994

Dirección de los autores. Departamento de Biología Vegetal. Facultad de Ciencias. Universidad de Málaga. Apdo. 59. 29080, Málaga. Fax. (95) 21319 44. 